\title{
The chemically peculiar double-lined spectroscopic binary HD $90264^{\star}$
}

\author{
C. Quiroga, A. F. Torres, and L. S. Cidale
}

\author{
Departamento de Espectroscopía, Facultad de Ciencias Astronómicas y Geofísicas, Universidad Nacional de La Plata, \\ Paseo del Bosque S/N, B1900FWA La Plata, Buenos Aires, Argentina \\ Instituto de Astrofísica de La Plata (CCT La Plata - CONICET, UNLP), Paseo del Bosque S/N, B1900FWA La Plata, Buenos Aires, \\ Argentina \\ e-mail: cquiroga@fcaglp.fcaglp.unlp.edu.ar
}

Received 27 March 2010 / Accepted 21 July 2010

\begin{abstract}
Context. HD 90264 is a chemically peculiar (CP) double-lined spectroscopic binary system of the type He-weak. Double-lined binaries are unique sources of data for stellar masses, physical properties, and evolutionary aspects of stars. Therefore, the determination of orbital elements is of great importance to study how the physical characteristics of CP stars are affected by a companion. Aims. We carried out a detailed spectral and polarimetric study of the spectroscopic binary system HD 90264 to characterize its orbit, determine the stellar masses, and investigate the spectral variability and possible polarization of the binary components.

Methods. We employed medium-resolution échelle spectra and polarimetric data obtained at the 2.15-m telescope at CASLEO Observatory, Argentina. We measured radial velocities and line equivalent widths with IRAF packages. The radial velocity curves of both binary components were obtained combining radial velocity data derived from the single line of $\mathrm{Hg}$ II $\lambda 3984 \AA$ and the double lines of Mg II $\lambda 4481 \AA$ A. Polarimetric data were studied by means of the statistical method of Clarke \& Stewart and the Welch test. Results. We found that both components of the binary system are chemically peculiar stars, deficient in helium, where the primary is a $\mathrm{He}$ variable and the secondary is a $\mathrm{Hg}-\mathrm{Mn}$ star. We derived for the first time the orbital parameters of the binary system. We found that the system has a quasi-circular orbit $(e \sim 0.04)$ with an orbital period of 15.727 days. Taking into account the circular orbit solution, we derived a mass ratio of $q=M_{\mathrm{He}-\mathrm{w}} / M_{\mathrm{Hg}-\mathrm{Mn}}=1.22$. We also found a rotational period of around 15-16 days, suggesting a spin-orbit synchronization. Possible signs of intrinsic polarization have also been detected.

Conclusions. HD 90264 is the first known binary system comprised of a He variable star as the primary component and a $\mathrm{Hg}-\mathrm{Mn}$ star as the secondary one.
\end{abstract}

Key words. binaries: spectroscopic - stars: chemically peculiar - stars: individual: HD 90264

\section{Introduction}

Among the upper-main-sequence chemically peculiar (CP) stars are the generally so-called Ap and Bp stars. These stars show slow rotation and abnormal abundance enhancements of some chemical species in their atmospheres as compared to "normal" A and B dwarfs of the same effective temperature (Jascheck \& Jascheck 1987). According to their chemical peculiarity the stars are classified in the following subgroups (Preston 1974): CP1 (metallic line stars, earlier designation Am, with overabundance of metals), CP2 (overabundance of $\mathrm{Si}, \mathrm{Cr}$, and $\mathrm{Sr}-\mathrm{Cr}-\mathrm{Eu}$, earlier known as Ap stars), CP3 (overabundance of $\mathrm{Hg}$ and/or $\mathrm{Mn}$ ) and CP4 (He-weak, B-type objects with abnormally weak lines of He I). In addition, Preston (1974) clearly distinguished two main groups: a non-magnetic sequence of $\mathrm{Am}$ and $\mathrm{Hg}$ $\mathrm{Mn}$ stars and a magnetic sequence of Ap and some He-weak stars. On the other hand, a large fraction of these stars are known to be binaries: the $\mathrm{CP} 1$ subgroup has $32.5 \%$ binaries and the $\mathrm{CP} 2$ group has $66 \%$ binaries with large magnetic fields (Seggewiss 1993), while more than $67.7 \%$ of the CP3 stars are members of

* Based on observations taken at Complejo Astronómico El Leoncito (CASLEO), operated under an agreement between the Consejo Nacional de Investigaciones Científicas y Técnicas de la República Argentina, the Secretaría de Ciencia y Tecnología de la Nación and the National Universities of La Plata, Córdoba and San Juan. binary systems (or of systems of higher multiplicity) (Hubrig \& Mathys 1995, 1996). Based on the information of 45 binary systems with known orbital periods, Gerbaldi et al. (1985) found a deficiency of binaries for the He-weak stars, Si stars, Si-Cr, and $\mathrm{Si}-\mathrm{Sr}$ stars and also confirmed a predominance of doublelined binaries (SB2) among Hg-Mn stars and a total absence of $\mathrm{SB} 2$ systems among the $\mathrm{Si}$ ones. This study also revealed a great deficiency among low-eccentricity systems for all peculiar stars except for the Hg-Mn ones, which was confirmed by Leone \& Catanzaro (1999).

The slow rotational velocity is generally accepted as a more direct reason for the $\mathrm{CP}$ phenomenon, but the origin of the braking mechanism is not clearly established. The most attractive theories are magnetic braking and orbital synchronization. Evidence in favor or against both hypotheses were widely discussed by Abt $(1965,1979)$ and Wolff $(1983)$.

The occurrence of CP stars in spectroscopic binaries might provide intriguing information about a possible connection between rotation, magnetism, and abundance anomalies (Budaj 1999; Noels et al. 2004; Hubrig et al. 2006a). The interaction of the companions not only influences the evolution of the system and changes the chemical composition of their atmospheres but also determines the internal rotation properties of the stars. In particular, double-lined binaries are unique sources of data for stellar masses, physical properties, and evolutionary aspects 
of stars. Furthermore, the determination of orbital elements is of great importance to study how the physical characteristics of $\mathrm{CP}$ stars are affected by a companion.

The aim of this research is to study HD 90264 in detail, which seems to be the only He-weak star ever reported to belong to a SB2 system (Pedersen \& Thomsen 1977). We here present spectroscopic data that allowed us for the first time to derive the orbital parameters, the stellar masses, and the spectral variability. Polarimetric data are also reported.

\subsection{The star}

The source HD $90264\left(=\right.$ HR $4089=$ CPD -66 1243; $\alpha_{2000}=$ $\left.10^{\mathrm{h}} 22^{\mathrm{m}} 58^{\mathrm{s}}, \delta_{2000}=-66^{\circ} 54^{\prime} 05^{\prime \prime}\right)$ is a bright $\left(m_{V}=4.95\right)$ B9 V star, member of the Sco-Cen Complex in the region of the Lower Centaurus Crux association (de Zeeuw et al. 1999). Jaschek et al. (1969) classified it as a chemically peculiar star belonging to the He-weak group due to a disagreement between the observed spectral type (B9) and the one inferred from the $U B V$ photometry (B6). Pedersen \& Thomsen (1977) found it to be a spectroscopic binary showing two spectra and identified it as a possible He variable. They determined three periods, $P=$ $0.9445,1.0565,17.7$ days, which reproduced the variation in the photometric R-index equally well. The star exhibits an IR excess of $28 \%$ (Groote \& Kaufmann 1981) and a moderate enhancement of the photoelectric parameter $\Delta a$-index (Maitzen 1981), which measures the strength of the $\lambda 5200 \AA$ flux depression.

Hubrig \& Mathys (1996) reported that HD 90264 showed a strong feature of $\mathrm{Hg}$ II $\lambda 3984 \AA$ in one of the binary components and proposed that the star might rather belong to the group of the P-Ga He-weak stars, hotter analogs of the nonmagnetic $\mathrm{Hg}-\mathrm{Mn}$ stars (Borra et al. 1983), than be related to the magnetic Si stars.

Circularly polarized spectra measurements of HD 90264, obtained with the focal reducer and low-dispersion spectrograph (FORS1) at the Very Large Telescope (VLT) of the European Southern Observatory (ESO) revealed a weak mean longitudinal magnetic field $\left\langle B_{1}\right\rangle$ of $114 \pm 108 \mathrm{G}$ (Hubrig et al. 2006b).

Fundamental parameters of this star such as absolute magnitude $\left(M_{\mathrm{V}}=-0.403\right)$, distance from the sun $(d=117.5 \mathrm{pc})$, effective temperature $\left(T_{\text {eff }}=15300 \mathrm{~K}\right)$ and age $\left(t=18 \times 10^{6}\right.$ years $)$ were published by Westin (1985), who included HD 90264 in a study of the distribution and kinematics of early-type stars. On the other hand, Gomez et al. (1998) reported $M_{\mathrm{V}}=-0.8$ and $d=$ 134 pc. In addition, Hunger \& Groote (1999) estimated for this star a $T_{\text {eff }}=14600 \mathrm{~K}$, a surface gravity $\log g=4.04$, an age of $76 \times 10^{6}$ years, and a mass $M_{*}=4.3 M_{\odot}$, when studying the $\mathrm{H} / \mathrm{He}$ abundance anomalies in B-type stars as a consequence of the existence of fractionated stellar winds.

Based on line profile fitting of Fe II lines, Dolk et al. (2003) estimated a projected rotational velocity $V \sin i=7 \mathrm{~km} \mathrm{~s}^{-1}$, which is one order of magnitude lower than the $79 \mathrm{~km} \mathrm{~s}^{-1}$ given in the Bright Star Catalogue.

\section{Observations}

High-resolution spectroscopic observations were carried out with the 2.15-m Jorge Sahade telescope at Complejo Astronómico el Leoncito (CASLEO), San Juan, Argentina, during six observing runs between 2005 and 2008. We used a REOSC échelle spectrograph in cross-dispersion mode with a Tek $1024 \times 1024$ pixel CCD as detector. A grating of 400 lines $\mathrm{mm}^{-1}$ was used except for the observing run in
May 2008, where we chose a grating of 316 lines $\mathrm{mm}^{-1}$. The covered wavelength ranges were $\lambda \lambda 3500-6000 \AA$ and $\lambda \lambda 3900-7100 \AA$, respectively. The signal-to-noise ratio $(\mathrm{S} / \mathrm{N})$ of the spectra was higher than 100 and the selected instrumental configuration yielded a resolving power, $R$, of 13000 in the blue region of the spectrum. A Th-Ar lamp was used as a comparison source, with a reference exposure taken immediately after each stellar target at the same telescope position. Spectral CCD images were reduced with IRAF $^{1}$ packages, CCDRED and ECHELLE, and all spectra were measured with the SPLOT task. Table 1, Cols. 1 and 2, shows the log of observations.

Polarimetric data were also obtained in April and June, 2007, with the photopolarimeter of Turin Observatory attached at the 2.15-m telescope of CASLEO. The instrument can take simultaneous measurements in five channels with color bands close to those of the standard UBVRI system, based on the design principles of Piirola $(1973,1988)$. The final response of the five photomultipliers corresponds to equivalent wavelengths of $0.36,0.43$, $0.53,0.65$ and $0.86 \mu \mathrm{m}$. During each observing run a set of zero and highly polarized standard stars were observed to determine the instrumental polarization and the coordinate transformation of the polarization position angle into the equatorial system, respectively. We subtracted the instrumental polarization from all data, which is typically of $\sim 0.01 \%$.

\subsection{The line spectrum}

The star HD 90264 displays a rich absorption sharp-lined spectrum. In addition to the typical lines of H I and He I, it is possible to distinguish lines of single-ionized elements such as $\mathrm{Mg}$ II, Mn II, Hg II, Si II, P II, and Fe II.

The double-lined nature of the star is clearly seen in most of the lines, while the transition at $\lambda 3984 \AA$ appears as a singlelined feature (see Fig. 1, left). We identified this transition as caused by $\mathrm{Hg}$ II and not Mn II, because the Mn II lines show double-lined features. Therefore, one of the binary components is a chemically peculiar star of $\mathrm{Hg}-\mathrm{Mn}$ and hereafter we will use the line of $\mathrm{Hg}$ II $\lambda 3984 \AA$ to identify this star. We were not able to find either $\operatorname{Hg}$ I $\lambda 4358 \AA$ or Pt II $\lambda 4046.45 \AA$ (the latter is closely blended with the $\mathrm{Hg}$ I $\lambda 4046.56 \AA$, which is also not observed). We also searched for $\mathrm{Hg}$ II $\lambda 6149 \AA$, but were not able to find it probably due to a blend with Fe II lines. Numerous lines of P II were found, but not Ga II. While Sr II $\lambda 4077 \AA$ is absent, Sr II $\lambda 4215 \AA$ might be among other very weak lines. The Si II $\lambda \lambda 3853,3856,3862,4128$, and $4130 \AA$ are well identified, but they are not enhanced.

The radial velocity (RV) values were obtained by a Gaussian fit of line profiles, except for the HI lines, for which we measured the barycentric wavelength of the profiles. Owing to the double component nature and numerous absorption lines, it was hard to perform a confident line-identification. Therefore, to avoid ambiguous or wrong identification of chemical elements, we first calculated the spectroscopic orbit of the $\mathrm{Hg}-\mathrm{Mn}$ star (see details in Sect. 3), traced by the single line of Hg II $\lambda 3984 \AA$ and then checked if the measured RV of other individual lines agreed with the RV curve computed previously. In Table 1 we quote the orbital phase and report our measurements of RVs for $\mathrm{Hg}$ II $\lambda 3984 \AA$ and $\mathrm{Mg}$ II $\lambda 4481 \AA$ derived

\footnotetext{
1 IRAF is distributed by the National Optical Astronomy Observatories, which is operated by the Association of Universities for Research in Astronomy (AURA) under cooperative agreement with the National Science Foundation.
} 

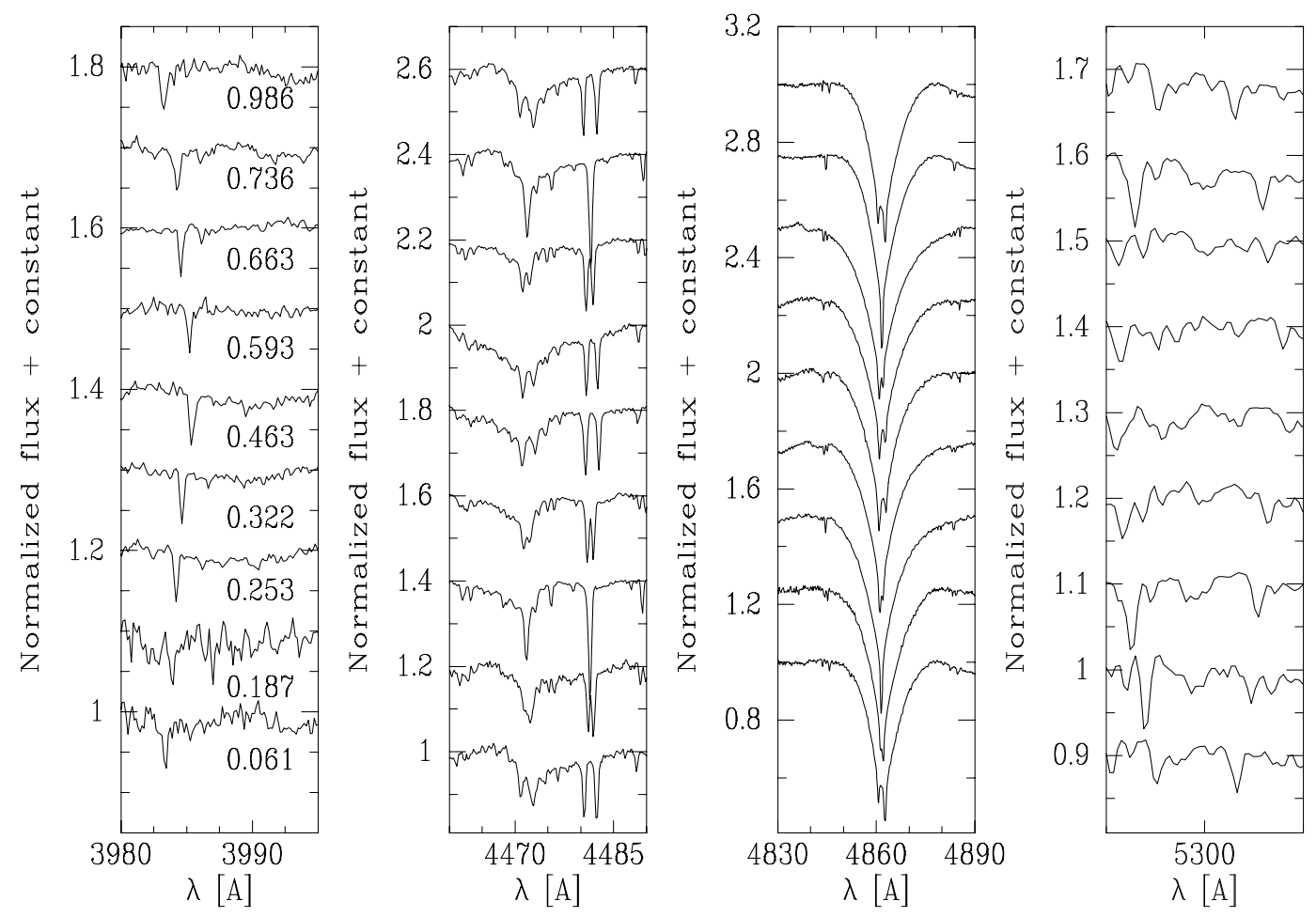

Fig. 1. Sample of the variation of the line profiles of $\mathrm{Hg}$ II $\lambda 3984 \AA$ (left), He I $\lambda 4471 \AA$ and Mg II $\lambda 4481 \AA$ (center-left), H $\beta$ (center-right) and Mn II $\lambda 5302 \AA$ (right) with the orbital phase.

from our spectroscopic observations and spectra taken from the ESO Science Archive Catalog (http://vizier.u-strasbg. fr) and Nitschelm (2004).

Table 1 also lists our measurements of the equivalent width $(E W)$ of the $\mathrm{Hg}$ II $\lambda 3984 \AA$ line and the $E W$ s derived from some lines observed in the spectra of both stars: $\mathrm{H} \beta$, He I $\lambda 4471 \AA$, Mg II $\lambda 4481 \AA$, Si II $\lambda 4130 \AA$, and Mn II $\lambda 5302 \AA$. All these lines are observed in absorption. A sample of the variation of the line profiles (Hg II $\lambda 3984 \AA$, He I $\lambda 4471 \AA$, Mg II $\lambda 4481 \AA, \mathrm{H} \beta$ and Mn II $\lambda 5302 \AA$ ) with the orbital phase is displayed in Fig. 1 . Except for the He I and $\mathrm{HI}$ lines, all absorption lines could be easily deconvolved into their stellar components. The line intensity of both stars is almost the same, pointing at a similar spectral type, although the lines of Mn II are more intense in the $\mathrm{Hg}-\mathrm{Mn}$ star. The measured EWs of He I lines of both stars are smaller than expected for a B8 V star (Didelon 1982), indicating that they present a deficiency in helium. We also noted that the $E W$ of He I $\lambda 4471 \AA$ line, which is not related to the $\mathrm{Hg}-\mathrm{Mn}$ star, is variable (see Table 1, Col. 10), thus confirming the helium variable nature of this object (Pedersen \& Thomsen 1977). We will identify this star as the He-w star.

We estimated an error of $10 \%$ in our measurements for the $E W$ and of $5 \mathrm{~km} \mathrm{~s}^{-1}$ for the RV. The accuracy in our measurements is mainly limited by the instrumental resolution, the $\mathrm{S} / \mathrm{N}$ ratio of our spectra, and the continuum determination.

\section{The spectroscopic orbits}

Although HD 90264 is a bright double-lined spectroscopic binary, its orbital parameters were not computed until now.

In order to obtain the orbital parameters, we minimized the weighted sum $\sum_{i=1}^{N} w_{i}(\mathrm{O}-\mathrm{C})_{i}^{2}$, where $\mathrm{O}$ and $\mathrm{C}$ are the observed and computed $\mathrm{RV}$ values, respectively, $w$ are the corresponding weights and $N$ is the number of data points. Because all data have the same quality, we took the same weight for all of them. The best circular orbital solution obtained from the RVs of Hg II $\lambda 3984 \AA$ gives a period of $P=15.7260 \pm 0.0015$ days. Later, we computed a new orbital solution combining the RVs of $\mathrm{Hg}$ II $\lambda 3984 \AA$ with those of Mg II $\lambda 4481 \AA$ to obtain the RV curves of both stars. In this case, a slightly different period of $P=15.7275 \pm 0.0008$ days was found. We calculated both circular and eccentric orbits. Table 2 lists the obtained orbital solutions. The notation has the usual meaning: $\gamma$ is the barycentric velocity, $K$ is the orbital semi-amplitude, $e$ is the eccentricity, $\omega$ is the longitude of the periastron, $T_{0}$ is the time of maximum velocity for circular orbits and the time of periastron passage for eccentric orbits. The elliptical orbit gives a slightly better solution than the circular one and, according to the Lucy \& Sweeney (1971) test, the value found for the eccentricity is significant. In Fig. 2 we show the obtained orbital solutions for circular and eccentric orbits. However, as the obtained eccentricity $e=$ $0.044 \pm 0.014$ is very low, the orbit is almost circular.

Thus, considering the orbital solution derived from a circular orbit, we obtained the semi-major axis sizes, which are $a_{\mathrm{He}-\mathrm{w}} \sin i=0.089 \pm 0.002 \mathrm{AU}$ and $a_{\mathrm{Hg}-\mathrm{Mn}} \sin i=0.109 \pm$ $0.002 \mathrm{AU}$ for the primary and secondary binary components, respectively. In addition, combining the semi-amplitudes of both stars (Table 2), we derived a mass ratio $q=M_{\mathrm{He}-\mathrm{w}} / M_{\mathrm{Hg}-\mathrm{Mn}}=$ $1.22 \pm 0.05$, which implies stellar masses of $M_{\mathrm{He}-\mathrm{w}} \sin ^{3} i=$ $2.3 \pm 0.1 M_{\odot}$ for the primary component and $M_{\mathrm{Hg}-\mathrm{Mn}} \sin ^{3} i=$ $1.9 \pm 0.1 M_{\odot}$ for the secondary. Then, assuming that the primary star has a stellar mass of $4.3 M_{\odot}$ (Hunger \& Groote 1999), we determined a stellar mass for the companion of $M_{\mathrm{Hg}-\mathrm{Mn}} \sim$ $3.5 M_{\odot}$, which is close to the expected value for late B-type dwarves, namely $3.8 M_{\odot}$ for a B8-type star (Cox 2000). We also obtained an inclination angle for the orbital plane of the binary system of $i \sim 54^{\circ}$. This value is consistent with the photometric 
A\&A 521, A75 (2010)

Table 1. Log of observations with their corresponding orbital phase; measurements of radial velocities (RVs) and line equivalent widths (EWs).

\begin{tabular}{|c|c|c|c|c|c|c|c|c|c|c|c|c|c|c|c|}
\hline Date & $\begin{array}{c}\text { JD } \\
2400000+\end{array}$ & Phase & \begin{tabular}{|c|} 
RV \\
Hg II $\lambda 3984$
\end{tabular} & $\begin{array}{l}\left.\mathrm{km} \mathrm{s}^{-1}\right] \\
\mathrm{Mg} \text { II } \lambda \\
\mathrm{Hg}-\mathrm{Mn}\end{array}$ & $\begin{array}{l}24481 \\
\text { He-w }\end{array}$ & Hg II $\lambda 3984$ & $\mathrm{H} \beta$ & $\begin{array}{c}\mathrm{He} \text { I } \lambda \\
\text { Hg-Mn }\end{array}$ & $\begin{array}{l}4471 \\
\text { He-w }\end{array}$ & 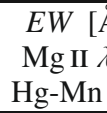 & $\begin{array}{l}\stackrel{8}{8}] \\
14481 \\
\text { He-w }\end{array}$ & \multicolumn{2}{|c|}{ Si II $\lambda 4130$} & \multicolumn{2}{|c|}{ Mn II $\lambda 5302$} \\
\hline $2002-02-26$ & $52331.241^{a}$ & 0.640 & 48.6 & 48.2 & -13.8 & - & - & - & - & - & - & - & - & - & - \\
\hline 2003-07-04 & $52825.6 . .^{b}$ & 0.073 & - & -52.2 & 87.1 & - & - & - & - & - & - & - & - & - & - \\
\hline 2003-07-07 & $52828.6 . .^{b}$ & 0.265 & - & 5.4 & 36.9 & - & - & - & - & - & - & - & - & - & - \\
\hline $2005-03-28$ & 53457.541 & $0.253^{c}$ & 27.8 & 24.8 & 24.8 & 0.03 & 5.92 & 0.21 & 0.21 & 0.19 & 0.19 & 0.08 & 0.08 & 0.03 & 0.03 \\
\hline $2005-03-28$ & 53457.610 & $0.257^{c}$ & 26.6 & 24.1 & 24.1 & 0.03 & 5.76 & 0.20 & 0.20 & 0.15 & 0.15 & 0.08 & 0.08 & 0.02 & 0.02 \\
\hline $2005-03-28$ & 53457.701 & $0.263^{c}$ & 30.4 & 25.4 & 25.4 & 0.02 & 5.26 & 0.20 & 0.20 & 0.19 & 0.19 & 0.08 & 0.08 & 0.03 & 0.03 \\
\hline 2005-03-29 & 53458.567 & 0.318 & 57.8 & 57.1 & -1.8 & 0.03 & 5.48 & 0.11 & 0.13 & 0.12 & 0.12 & 0.03 & 0.05 & 0.03 & 0.01 \\
\hline 2005-03-29 & 53458.630 & 0.322 & 58.2 & 57.0 & -2.2 & 0.03 & 5.50 & 0.11 & 0.13 & 0.10 & 0.11 & 0.03 & 0.04 & 0.03 & 0.01 \\
\hline 2005-03-29 & 53458.703 & 0.327 & 59.5 & 58.2 & -5.4 & 0.03 & 5.85 & 0.07 & 0.19 & 0.09 & 0.10 & 0.03 & 0.05 & 0.02 & 0.01 \\
\hline 2005-06-17 & 53539.479 & 0.463 & 95.6 & 99.1 & -37.5 & 0.02 & 5.71 & 0.12 & 0.21 & 0.09 & 0.10 & 0.03 & 0.05 & 0.03 & 0.02 \\
\hline 2005-06-17 & 53539.484 & 0.463 & 99.1 & 98.1 & -36.3 & 0.03 & 5.28 & 0.11 & 0.21 & 0.09 & 0.10 & 0.03 & 0.05 & 0.03 & 0.02 \\
\hline 2005-06-19 & 53541.481 & 0.590 & 87.4 & 85.1 & -31.0 & 0.02 & 5.18 & 0.13 & 0.16 & 0.10 & 0.10 & 0.03 & 0.04 & & \\
\hline 2005-06-20 & 53541.524 & 0.593 & 89.7 & 85.2 & -27.0 & 0.02 & 5.46 & 0.16 & 0.18 & 0.09 & 0.10 & 0.03 & 0.05 & 0.02 & 0.02 \\
\hline 2007-01-08 & 54108.820 & 0.663 & 61.4 & 62.3 & -8.5 & 0.02 & 5.89 & 0.11 & 0.15 & 0.09 & 0.11 & 0.03 & 0.05 & 0.02 & 0.01 \\
\hline 2007-04-27 & 54218.464 & 0.634 & 70.5 & 72.4 & -17.8 & 0.02 & 5.39 & 0.10 & 0.17 & 0.09 & 0.12 & 0.03 & 0.05 & 0.02 & 0.01 \\
\hline 2007-06-10 & 54261.503 & 0.371 & 80.3 & 79.3 & -15.4 & 0.02 & 5.52 & 0.08 & 0.14 & 0.09 & 0.11 & 0.03 & 0.05 & 0.02 & 0.01 \\
\hline 2008-05-11 & 54597.519 & $0.736^{c}$ & 23.4 & 22.7 & 22.7 & 0.03 & 4.68 & 0.25 & 0.25 & 0.18 & 0.18 & 0.08 & 0.08 & 0.03 & 0.03 \\
\hline $2008-05-13$ & 54600.472 & 0.924 & -50.0 & -42.4 & 78.9 & 0.04 & 4.45 & 0.10 & 0.22 & 0.07 & 0.10 & 0.04 & 0.05 & 0.03 & 0.01 \\
\hline $2008-05-14$ & 54601.455 & 0.986 & -54.1 & -52.5 & 83.5 & 0.03 & 4.71 & 0.13 & 0.23 & 0.08 & 0.10 & 0.05 & 0.05 & 0.03 & 0.01 \\
\hline 2008-05-15 & 54602.453 & 0.049 & -57.1 & -49.7 & 80.8 & 0.04 & 4.89 & 0.13 & 0.28 & 0.08 & 0.10 & 0.05 & 0.06 & 0.02 & 0.01 \\
\hline 2008-05-16 & 54602.629 & 0.061 & -44.3 & -47.3 & 83.2 & 0.03 & 4.95 & 0.10 & 0.23 & 0.08 & 0.10 & 0.05 & 0.05 & 0.03 & 0.02 \\
\hline $2008-05-17$ & 54603.553 & 0.119 & -32.6 & -33.3 & 69.8 & 0.04 & 4.80 & 0.12 & 0.18 & 0.07 & 0.09 & 0.04 & 0.06 & 0.02 & 0.01 \\
\hline $2008-05-18$ & 54604.624 & 0.187 & -2.5 & -4.3 & 48.5 & 0.04 & 5.37 & 0.13 & 0.15 & 0.08 & 0.10 & 0.03 & 0.05 & 0.02 & 0.01 \\
\hline $2008-05-18$ & 54605.457 & $0.240^{c}$ & 9.5 & 23.0 & 23.0 & 0.04 & 4.98 & 0.21 & 0.21 & 0.19 & 0.19 & 0.08 & 0.08 & 0.02 & 0.02 \\
\hline 2008-05-19 & 54605.545 & $0.246^{c}$ & 11.4 & 22.9 & 22.9 & 0.03 & 5.26 & 0.20 & 0.20 & 0.18 & 0.18 & 0.09 & 0.09 & 0.06 & 0.06 \\
\hline $2008-05-20$ & 54606.594 & 0.313 & 42.8 & 53.5 & 0.7 & 0.02 & 4.88 & 0.13 & 0.10 & 0.07 & 0.10 & 0.02 & 0.06 & 0.02 & 0.01 \\
\hline
\end{tabular}

Notes. Because the $\mathrm{H} \beta$ feature could not be deconvolved into its stellar components, the $E W$ quoted in the table corresponds to the total value. ${ }^{(a)}$ ESO Science Archive Catalog (Vizier); ${ }^{(b)}$ from Nitschelm (2004); ${ }^{(c)}$ these phases are near conjunction, where HD 90264 shows a single-lined spectrum. For those lines that are present in both stars, the RV and line- $E W$ values measured in these single-lined spectra were repeated.

Table 2. Orbital solutions for HD 90264.

\begin{tabular}{cccccccc}
\hline \hline & $\begin{array}{c}P \\
{[\text { days }]}\end{array}$ & $\begin{array}{c}\gamma \\
{\left[\mathrm{km} \mathrm{s}^{-1}\right]}\end{array}$ & $\begin{array}{c}K_{\mathrm{He}-\mathrm{w}} \\
{\left[\mathrm{km} \mathrm{s}^{-1}\right]}\end{array}$ & $\begin{array}{c}K_{\mathrm{Hg}-\mathrm{Mn}} \\
{\left[\mathrm{km} \mathrm{s}^{-1}\right]}\end{array}$ & $e$ & $\omega$ & $\begin{array}{c}T_{0}{ }^{a} \\
{[\mathrm{JD}]}\end{array}$ \\
\hline Hg II & $15.726 \pm 0.001$ & $22.2 \pm 1.0$ & - & $78.9 \pm 1.4$ & $0^{b}$ & - & $2453540.00 \pm 0.04$ \\
Hg II+Mg II & $15.727 \pm 0.001$ & $22.5 \pm 0.8$ & $61.8 \pm 2.0$ & $75.6 \pm 1.4$ & $0^{b}$ & - & $2452824.46 \pm 0.07$ \\
Hg II+Mg II & $15.727 \pm 0.001$ & $22.7 \pm 0.7$ & $62.2 \pm 1.9$ & $76.0 \pm 1.5$ & $0.044 \pm 0.014$ & $138^{\circ} \pm 25^{\circ}$ & $2452814.78 \pm 1.05$ \\
\hline
\end{tabular}

Notes. ${ }^{(a)}$ Time of maximum velocity for circular orbit and time of periastron passage for eccentric orbit; ${ }^{(b)}$ assumed value to obtain the orbital solution.

observations obtained by the Hipparcos satellite $\left(H_{\mathrm{p}}=4.9278 \pm\right.$ 0.0004, Adelman 2001), which present no evidence of eclipses.

\section{Spectral variability}

In Figs. 3 and 4 we show the variability of the EWs of some absorption lines with the orbital period, and below we describe the behavior of each line transition.

1. $\mathrm{H} \beta$ (Fig. 3, top-left): because it was not possible to separate the line profile of each star, we present the variation of the total $E W$ with the orbital phase. We observed that the $E W$ increases by a factor of 1.5 from phases around 0 to 0.5 . We were not able to find confident periods shorter than the orbital one, instead we obtained two possible periods of 36.0 and 25.0 days. We might attribute these large periods to the effect of line intensity variations in both stars.

2. Hg II $\lambda 3984 \AA$ (Fig. 3, bottom-left): this line is only observed in the $\mathrm{Hg}-\mathrm{Mn}$ star. It shows slight intensity variations with the orbital period with a minimum at around phase 0.5 . The best period found for the $E W$ data is 15.2 days, which is very close to the orbital one.

3. Mg II $\lambda 4481 \AA$ (Fig. 3, top-right): both line components remain constant with the orbital period. The intensity of the $\mathrm{Mg}$ line of the He-w star is slightly stronger than that of the $\mathrm{Hg}-\mathrm{Mn}$ one.

4. Mn II $\lambda 5302 \AA$ (Fig. 3, bottom-right): none of the line components shows line- $E W$ variations with the orbital period. The strongest line is seen in the $\mathrm{Hg}-\mathrm{Mn}$ star. We did not find emission features in the Mn II line profiles as for the He-weak star 3 Cen A and other Hg-Mn stars (Wahlgren \& Hubrig 2004).

5. Si II $\lambda 4130 \AA$ (Fig. 4, top): this line is clearly seen in both stars, and is strongest in the He-w star. The line displays small intensity variations with the orbital period in the $\mathrm{Hg}-\mathrm{Mn}$ star with a maximum at phase 0 . The best period that fits these data is 16.2 days, which is close to the orbital period. No variations are observed in the He-w star. The sum of 


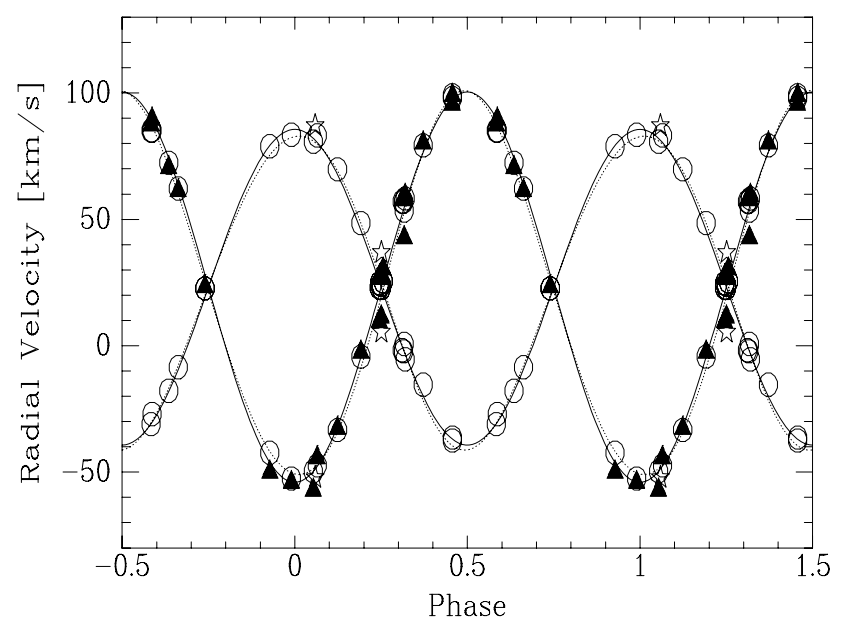

Fig. 2. Radial velocity data and orbital solutions for HD 90264. Open circles and filled triangles represent data from CASLEO (Mg II and $\mathrm{Hg}$ II, respectively). Star symbols correspond to data from Nitschelm (2004) and ESO/UVES Science Archive Catalog (Vizier). The solid and dotted lines trace the circular and eccentric orbits, respectively.
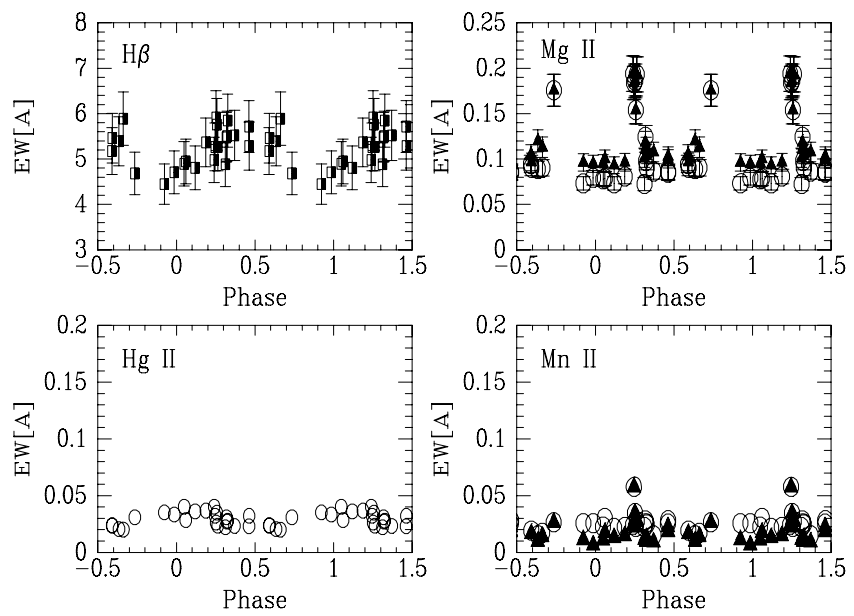

Fig. 3. Variation of the equivalent widths ( $E W \mathrm{~s}$ ) of $\mathrm{H} \beta$ and $\mathrm{Mg}$ II, $\mathrm{Hg}$ II and Mn II lines with the orbital phase. Semifilled squares (top-left) represent the sum of the equivalent widths of the $\mathrm{H} \beta$ line of both stars, open circles represent data related to the $\mathrm{Hg}-\mathrm{Mn}$ star, and filled triangles correspond to the He-w star. Error bars are omitted when they are less than the symbol size.

the line- $E W$ s of both stars phased with the orbital period are illustrated in the third plot to highlight the found variation.

6. He I $\lambda 4471 \AA$ (Fig. 4, bottom): the He I line of the Hg-Mn star is weaker than that of the He-w star. In addition, the line$E W$ of the $\mathrm{Hg}-\mathrm{Mn}$ star remains almost constant, while the He-w star shows line intensity variations by a factor of up to 3 between the maximum and minimum. Two maxima of different values are observed around phases 0 and 0.5 . The sum of the line- $E W \mathrm{~s}$ of both stars phased with the orbital period are depicted in the third plot to emphasize the found variation. We determined a periodic variability of 15.8 days, this value is also close to the orbital period (see Table 2).

\section{Polarimetry}

Table 3 lists for each night the observed polarimetric data in the $U B V R I$ bands in terms of the weighted mean values of the Stokes parameters $P X$ and $P Y$ (in percent terms), the corresponding degree of linear polarization $P(\%)$, the position angle PA (in degrees), and their corresponding mean errors $M E$. The number of observations $N$ is also indicated. To obtain the averaged values of $P(\%)$ and PA for each night, we used weights determined from the inverse square of the estimated error for each observation. The estimated error is taken either from the least-square fit of the double cosine curves to the eight integrations acquired at the different positions of the $\lambda / 2$ plate or from the photon statistics, whichever is greater (Piirola 1973, 1988).

The standard criterion to evaluate if a measurement is polarized to some degree, either from intrinsic or interstellar effects, involves a comparison of a determined value with an estimate of its uncertainty. However, the degree of the linear polarization is a biased estimator of the true polarization; partly due to the noise on the signal (photon noise or intensity scintillation), which introduces a partial coherence between the measurements of the Stokes parameters, and partly to the conversion to the equatorial frame, which carries errors of the axis transformation that may be systematically different at each wavelength. This introduces difficulties in the analysis of the data of low polarization or low signal-to-noise level. Because UBVRI polarization of HD 90264 is too low to claim from its mean errors that the binary system is polarized, we used the statistical method of Clarke \& Stewart (1986) to gain a confident detection. According to this method, we detected polarization at $99 \%$ confidence level in the $U, V$, and $R$ bands in April 2007. In June 2007, we found polarization at $95 \%$ confidence level only in the $B$ band. However, in none of the observing runs statistical polarization was detected in the I band. Unfortunately, most of the nights did not have good polarimetric conditions.

In addition, we looked for polarimetric variability by means of the Welch test, because "Welch procedures may result in a polarimetric difference being detected between sets of points when none of the individual points is polarized, because the Welch test weights observations with respect to mean values and standard mean errors" (Clarke \& Stewart 1986). From our data we only detected a night-to-night variability in the position angle of the $V$ band in June 2007 with a confidence level of $99 \%$. At this point, we should recall that our data are in the same orbital phase, thus no large variations are expected between both observing runs.

Figure 5 shows the behavior of the average of all polarimetric data $(P(\%), \mathrm{PA}, P X$, and $P Y)$ with the wavelength for all observing nights. The fit of our data with the Serkowski's law (Serkowski et al. 1975) for interstellar polarization is shown in a dashed line. Taking into account that a) Serkowski's law does not seem to fit our data well, although if the error bars were only slightly larger, the dashed line would be a reasonable fit; b) based on the stellar polarization data of the Heiles (2000) catalogs, the stars in the field of HD 90264 do not show a tendency to present interstellar polarization; c) there is a difference of $30^{\circ}$ in the position angle in the $B$ band in relation to the PA of the other bands; and d) there is a confident statistical detection of low polarization, we can conclude that HD 90264 might present a variable intrinsic polarization.

\section{Discussion and conclusions}

We made a study of the chemically peculiar star HD 90264 based on spectral and polarimetric data. This object displays a clear SB2 spectrum with both stars deficient in helium. The system consists of a He variable star and a Hg-Mn companion star in a quasi-circular orbit. Assuming a circular orbit 

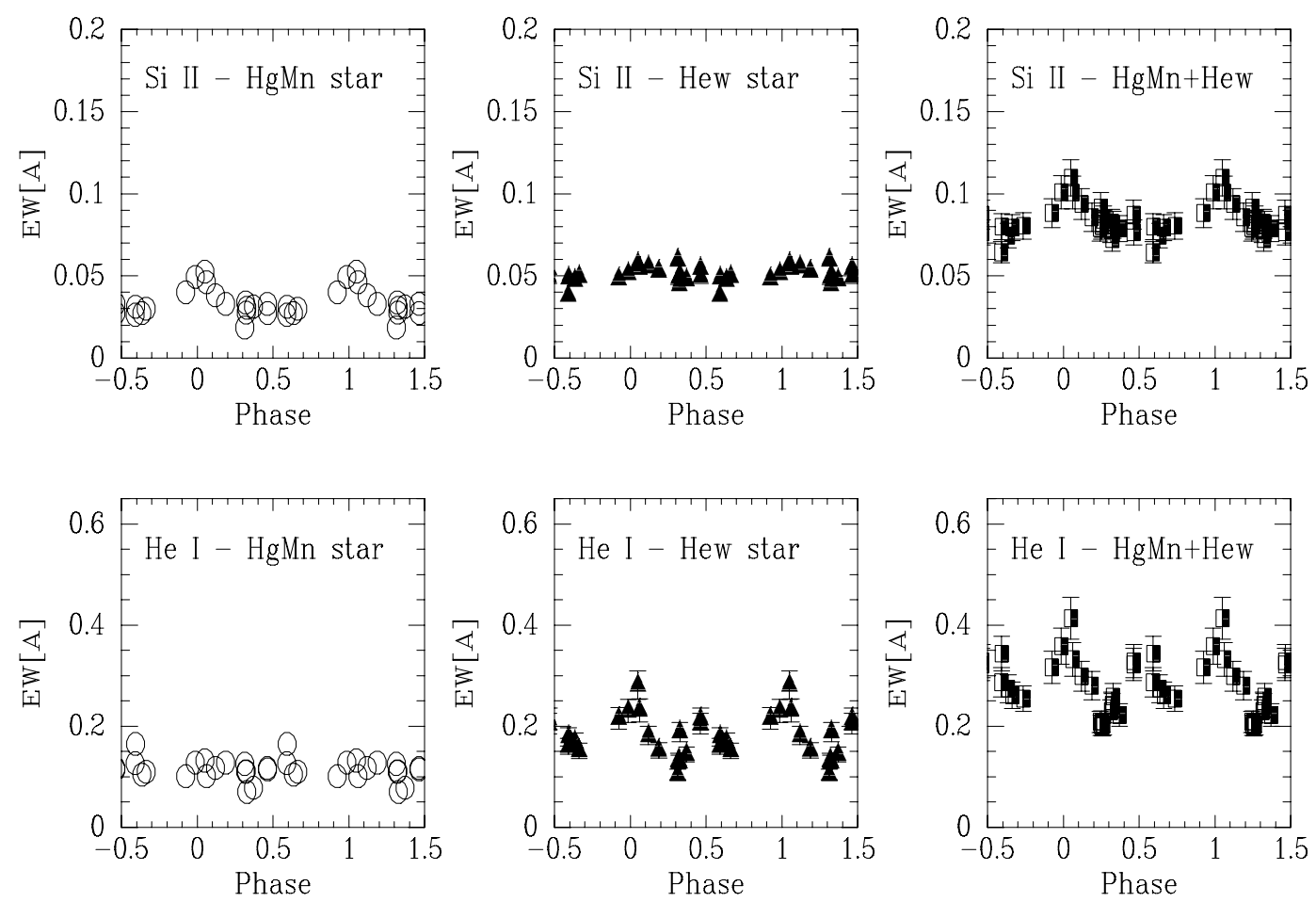

Fig. 4. Variation of the equivalent widths $(E W \mathrm{~s})$ of Si II and He I lines with the orbital phase: plots on the left and center illustrate the variation corresponding to each star, the plots on the right indicate the total contribution of both stars to the $E W$. Absence of error bars indicates that the magnitude of error is less than the symbol size.

Table 3. Polarimetric data of HD 90264.

\begin{tabular}{|c|c|c|c|c|c|c|c|c|c|c|}
\hline Date & JD-2 400000 & Phase & Band & $P X(\%)$ & $P Y(\%)$ & $P(\%)$ & $M E$ & $P A\left(^{\circ}\right)$ & $M E$ & $N$ \\
\hline \multirow[t]{5}{*}{$2007-04-24$} & 14215.479 & 0.445 & $U$ & -0.054 & -0.106 & 0.119 & 0.022 & 121.6 & 5.1 & 4 \\
\hline & & & $B$ & 0.032 & -0.038 & 0.049 & 0.023 & 155.0 & 12.5 & 4 \\
\hline & & & V & 0.021 & -0.223 & 0.224 & 0.040 & 137.7 & 5.0 & 4 \\
\hline & & & $R$ & 0.034 & -0.091 & 0.097 & 0.021 & 145.1 & 6.0 & 4 \\
\hline & & & I & 0.013 & -0.112 & 0.113 & 0.071 & 138.3 & 16.2 & 4 \\
\hline \multirow[t]{5}{*}{$2007-04-25$} & 14216.615 & 0.517 & $U$ & 0.021 & -0.064 & 0.067 & 0.030 & 144.2 & 12.0 & 4 \\
\hline & & & $B$ & 0.025 & -0.019 & 0.031 & 0.023 & 161.8 & 18.2 & 4 \\
\hline & & & V & 0.006 & -0.100 & 0.100 & 0.032 & 136.6 & 8.8 & 4 \\
\hline & & & $R$ & 0.005 & -0.084 & 0.084 & 0.021 & 136.7 & 6.8 & 4 \\
\hline & & & $I$ & 0.075 & -0.053 & 0.092 & 0.047 & 162.3 & 13.5 & 4 \\
\hline \multirow[t]{5}{*}{ 2007-06-10 } & 14262.471 & 0.433 & $U$ & 0.078 & -0.037 & 0.087 & 0.068 & 167.4 & 19.1 & 4 \\
\hline & & & $B$ & 0.270 & 0.210 & 0.342 & 0.167 & 199.0 & 13.0 & 4 \\
\hline & & & V & 0.375 & -0.054 & 0.379 & 0.149 & 175.9 & 10.7 & 4 \\
\hline & & & $R$ & 0.332 & -0.057 & 0.337 & 0.211 & 175.1 & 16.0 & 4 \\
\hline & & & $I$ & 0.480 & 0.097 & 0.490 & 0.160 & 5.7 & 9.1 & 4 \\
\hline \multirow[t]{5}{*}{ 2007-06-11 } & 14263.544 & 0.501 & $U$ & 0.080 & -0.034 & 0.087 & 0.042 & 168.3 & 12.9 & 4 \\
\hline & & & $B$ & 0.161 & -0.099 & 0.189 & 0.036 & 164.2 & 5.4 & 4 \\
\hline & & & V & -0.067 & -0.144 & 0.159 & 0.053 & 122.5 & 9.2 & 4 \\
\hline & & & $R$ & 0.121 & -0.031 & 0.125 & 0.043 & 172.9 & 9.6 & 4 \\
\hline & & & $I$ & -0.030 & -0.191 & 0.193 & 0.095 & 130.5 & 13.1 & 4 \\
\hline
\end{tabular}

solution, we derived for the first time the orbital parameters of HD 90264 and found an orbital period of $15.727 \pm 0.001$ days. The found orbital solution agrees with the statistical work of Gerbaldi et al. (1985) and Budaj (1999). In addition, we derived a mass ratio of $q=1.22 \pm 0.05$, which indicates that for the primary star $M_{\mathrm{He}-\mathrm{w}} \sin ^{3} i=2.3 \pm 0.1 M_{\odot}$ and for the secondary $M_{\mathrm{Hg}-\mathrm{Mn}} \sin ^{3} i=1.9 \pm 0.1 M_{\odot}$.

The He variable star displays variations in the He I $\lambda 4471 \AA$ line with a period of 15.8 days, reaching two maxima around phases 0 and 0.5 , which suggests the presence of two opposed enhanced helium abundance patches. Instead, the $\mathrm{Hg}-\mathrm{Mn}$ star shows variations in the $\mathrm{Hg}$ and $\mathrm{Si}$ lines with a period of about 15.2 and 16.2 days, respectively, and does not show variation in the He I $\lambda 4471$ line. The periods of the line variations observed in both stars are close to the orbital period. Variations in the $\mathrm{H} \beta$ line were also detected.

In the framework of the oblique rotator models, the variations of the observed line spectrum is related to the rotational period, and the observed spectral changes are explained as a consequence of a non-homogeneous emitting surface due to a non-uniform distribution of elements over the stellar surface. The periods found in the variations of the line equivalent widths 

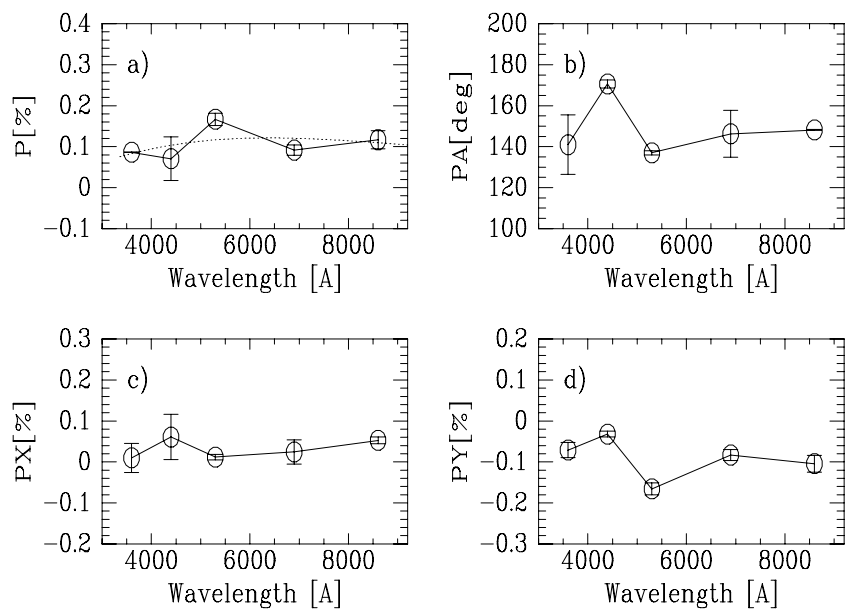

Fig. 5. Average of polarimetric data of HD 90264 with wavelength: a) polarization degree $P(\%)$, b) position angle PA, c) and d) Stokes parameters $P X$ and $P Y$, respectively. The dashed line in plot a) shows the fit of our data with the Serkowski's law for interstellar polarization.

of both stars are very close to the determined orbital period (15.727 days). This result suggests a spin-orbit synchronization in a quasi-circular orbit. Thus, assuming a 15.727 days variation in line intensity due to rotation, we found that the equatorial rotational velocity of the star is $11.6 \mathrm{~km} \mathrm{~s}^{-1}$. This value is consistent with the measurement of $V \sin i=7 \mathrm{~km} \mathrm{~s}^{-1}$ determined by Dolk et al. (2003), if we consider an inclination angle of $\sim 37^{\circ}$, a lower value than the one derived from the mass ratio.

We could also test the spin-orbit synchronization of HD 90264 with the Kitamura and Kondo test (Kitamura \& Kondo 1978). From the relation

$V \sin i=50.6 \frac{R_{\mathrm{sync}} \sin i}{P}$,

where $V \sin i$ is in $\mathrm{km} \mathrm{s}^{-1}, P$ is the orbital period in days, and $R_{\text {sync }}$ is the stellar radius, when synchronism has been reached, in solar radii, we obtained for $i=54^{\circ}, R_{\text {sync }} \sim 2.7 R_{\odot}$, and for $i=37^{\circ}, R_{\text {sync }} \sim 3.6 R_{\odot}$. Because an expected value for the radius of a B6-type star is $R_{*}=3.6 R_{\odot}$ (see Cox 2000), the necessary condition for synchronism, $R_{*} \geq R_{\text {sync }}$, is fulfilled in both cases.

In addition, we could estimate the spin-orbit synchronization time-scale of the system according to the two competing theories developed to explain the spin-orbit synchronization observed in detached closed binaries: the tidal theory of Zahn $(1975,1977)$ and the hydrodynamical theory of Tassoul (1987, 1988). We computed the characteristic synchronization time, in Zahn's case,

$t_{\text {sync }} \propto q^{2}\left(1+\frac{1}{q}\right)^{-5 / 6}\left(R_{*} / a\right)^{-17 / 2} \sim 2.77 \times 10^{9}$ years,

and in Tassoul's case,

$t_{\text {sync }} \propto q\left(1+\frac{1}{q}\right)^{-3 / 8}\left(R_{*} / a\right)^{-33 / 8} \sim 3.89 \times 10^{5}$ years,

where $q=M_{1} / M_{2}$ is the mass ratio of the system, $M_{1}$ and $M_{2}$ are the masses of the primary and secondary stars, respectively, $R_{*}$ is the radius of the primary star, and $a$ is the true mean separation of the binary components. For these calculations we also assumed the primary radius to be $3.6 R_{\odot}$, and computed the orbital separation $a \sim 52.66 R_{\odot}$ for $i=54^{\circ}$. The comparison between the obtained time-scales for the synchronization of the system with the estimated age of HD 90264, which is $\leq 7.6 \times 10^{7}$ years, would agree with the spin-orbit synchronization in the context of Tassoul's theory, which is based on the dissipation of the energy of large-scale meridional flows. If the inclination angle is smaller, the result is the same.

Concerning the polarimetric data, we found that HD 90264 might present a low level of intrinsic polarization. However, further observations are needed to confirm this result and the possible polarimetric variations with the orbital period of the binary system.

Acknowledgements. We thank our anonymous referee for the many comments and suggestions that helped to improve this manuscript. This research has made use of the VizieR catalogue access tool, CDS, Strasburg, France. L.C. acknowledges financial support from the Agencia de Promoción Científica y Tecnológica (Préstamo BID, PICT 111) and the Programa de Incentivos G11/089 of the Universidad Nacional de La Plata, Argentina.

\section{References}

Abt, H. A. 1965, ApJS, 11, 429

Abt, H. A. 1979, AJ, 84, 1591

Adelman, S. J. 2001, A\&A, 367, 297

Borra, E. F., Landstreet, J. D., \& Thompson, I. 1983, ApJS, 53, 151

Budaj, J. 1999, MNRAS, 310, 419

Clarke, D., \& Stewart, B. G. 1986, Vistas Astron., 29, 27

Cox, A. N. 2000, Allen's Astrophysical Quantities (New York: Springer-Verlag New York, Inc.)

de Zeeuw, P. T., Hoogerwerf, R., de Bruijne, J. H. J., Brown, A. G. A., \& Blaauw, A. 1999, AJ, 117, 354

Didelon, P. 1982, A\&AS, 50, 199

Dolk, L., Wahlgren, G. M., \& Hubrig, S. 2003, A\&A, 402, 299

Gerbaldi, M., Floquet, M., \& Hauck, B. 1985, A\&A, 146, 341

Gomez, A. E., Luri, X., Grenier, S., et al. 1998, A\&A, 336, 953

Groote, D., \& Kaufmann, J. P. 1981, A\&A, 94, L23

Heiles, C. 2000, AJ, 119, 923

Hubrig, S., \& Mathys, G. 1995, Comments Astrophys., 18, 167

Hubrig, S., \& Mathys, G. 1996, A\&AS, 120, 457

Hubrig, S., González, J. F., Savanov, I., et al. 2006a, MNRAS, 371, 1953

Hubrig, S., North, P., Schöller, M., \& Mathys, G. 2006b, Astron. Nachr., 327, 289

Hunger, K., \& Groote, D. 1999, A\&A, 351, 554

Jascheck, C., \& Jascheck, M. 1987, The Classification of Stars (Cambridge: Cambridge University Press)

Jaschek, M., Jaschek, C., \& Arnal, M. 1969, PASP, 81, 650

Kitamura, M., \& Kondo, M. 1978, Ap\&SS, 56, 341

Leone, F., \& Catanzaro, G. 1999, A\&A, 343, 273

Lucy, L. B., \& Sweeney, M. A. 1971, AJ, 76, 544

Maitzen, H. M. 1981, A\&A, 95, 213

Nitschelm, C. 2004, in Spectroscopically and Spatially Resolving the Components of the Close Binary Stars, ed. R. W. Hilditch, H. Hensberge, \& K. Pavlovski (San Francisco: ASP), ASP Conf. Ser., 318, 291

Noels, A., Montalbán, J., \& Maceroni, C. 2004, in The A-Star Puzzle, ed. J. Zverko, J. Ziznovsky, S. J. Adelman, \& W. W. Weiss (Cambridge: Cambridge University Press), IAU Symp., 224, 47

Pedersen, H., \& Thomsen, B. 1977, A\&AS, 30, 11

Piirola, V. 1973, A\&A, 27, 383

Piirola, V. 1988, in Polarized Radiation of Circumstellar Origin, ed. G. V. Coyne, A. M. Magalhaes, A. F. Moffat, R. E. Schulte-Ladbeck, \& S. Tapia (Vatican City: Vatican Press), 735

Preston, G. W. 1974, ARA\&A, 12, 257

Seggewiss, W. 1993, in IAU Colloq., 138, Peculiar versus Normal Phenomena in A-type and Related Stars, ed. M. M. Dworetsky, F. Castelli, \& R. Faraggiana (San Francisco: ASP), ASP Conf. Ser., 44, 137

Serkowski, K., Mathewson, D. S., \& Ford, V. L. 1975, ApJ, 196, 261

Tassoul, J. 1987, ApJ, 322, 856

Tassoul, J. 1988, ApJ, 324, L71

Wahlgren, G. M., \& Hubrig, S. 2004, A\&A, 418, 1073

Westin, T. N. G. 1985, A\&AS, 60, 99

Wolff, S. C. 1983, The A-stars: Problems and perspectives. Monograph series on nonthermal phenomena in stellar atmospheres (Washington, D.C.: NASA), SP-463

Zahn, J. 1975, A\&A, 41, 329

Zahn, J. 1977, A\&A, 57, 383 